\title{
Hypervelocity microparticle impact studies using a novel cosmic dust mass spectrometer
}

\author{
Daniel E. Austin, ${ }^{1,2}$ Ronald L. Grimm, ${ }^{1}$ Heidi L. K. Manning, ${ }^{3}$ Carl L. Bailey, ${ }^{3}$ \\ James E. Farnsworth, ${ }^{3}$ Thomas J. Ahrens, ${ }^{4}$ and J. L. Beauchamp ${ }^{1}$ \\ Received 5 June 2002; revised 27 February 2003; accepted 11 March 2003; published 14 May 2003.
}

[1] Micron-sized iron and copper particles accelerated to $2-20 \mathrm{~km} / \mathrm{s}$ in a $2 \mathrm{MV}$ van de Graaff electrostatic accelerator were used to test the performance of our recently developed cosmic dust mass spectrometer. This compact in situ dust analyzer, known as the Dustbuster, is designed to determine the elemental composition of cosmic dust particles through impact ionization and subsequent time-of-flight mass spectrometry. Results from 750 laboratory impacts show high mass resolution, typically $150-350(\mathrm{~m} / \Delta \mathrm{m})$ for projectile components and 300-600 for the target material (tantalum). Peaks corresponding to $\mathrm{H}, \mathrm{C}, \mathrm{O}, \mathrm{Na}$, and $\mathrm{K}$ ions are also observed, consistent with previous microparticle impact experiments. Fieldinduced emission of electrons immediately before impact is a possible cause of ion formation from species with high ionization potentials. The high mass resolution, large sensitive target area, and small size make the Dustbuster an ideal instrument for inclusion on a spacecraft payload. INDEX TERMS: 2129 Interplanetary Physics: Interplanetary dust; 6022 Planetology: Comets and Small Bodies: Impact phenomena; 6094 Planetology: Comets and Small Bodies: Instruments and techniques; 6297 Planetology: Solar System Objects: Instruments and techniques; 6215 Planetology: Solar System Objects: Extraterrestrial materials; KEYWORDS: Cosmic dust, impact ionization, hypervelocity impacts

Citation: Austin, D. E., R. L. Grimm, H. L. K. Manning, C. L. Bailey, J. E. Farnsworth, T. J. Ahrens, and J. L. Beauchamp, Hypervelocity microparticle impact studies using a novel cosmic dust mass spectrometer, J. Geophys. Res., 108(E5), 5038, doi:10.1029/2002JE001947, 2003.

\section{Introduction}

[2] In recent years considerable interest has arisen in cosmic dust, the fine-grained matter that pervades the solar system and the observable universe. Over the past four decades, several instruments have been developed and deployed on spacecraft to measure the size, trajectory, composition, and distribution of dust grains in the solar system. While most of these instruments study individual dust grains in situ [Grün, 1994; Srama and Grün, 1997], other methods have been developed to return dust samples to Earth for laboratory analysis [Brownlee et al., 1995].

[3] Spacecraft encounter dust grains at velocities ranging from a few to over a hundred $\mathrm{km} / \mathrm{s}$. At such speeds, an impacting dust grain and a portion of the surface it hits are partially vaporized and ionized [Hansen, 1968; Hornung and Kissel, 1994]. In situ chemical analyzers rely on this impact ionization, using mass spectrometric techniques to separate and measure the resulting ions on the basis of their

\footnotetext{
${ }^{1}$ Division of Chemistry and Chemical Engineering, California Institute of Technology, Pasadena, California, USA. USA.

${ }^{2}$ Now at Sandia National Laboratories, Albuquerque, New Mexico, USA.

${ }^{3}$ Department of Physics, Concordia College, Moorhead, Minnesota,

${ }^{4}$ Division of Geological and Planetary Sciences, California Institute of Technology, Pasadena, California, USA.

Copyright 2003 by the American Geophysical Union. 0148-0227/03/2002JE001947
}

atomic or molecular masses. Instruments of this type include the PIA and PUMA dust analyzers that encountered Halley's comet [Kissel et al., 1986], the Cassini Cosmic Dust Analyzer (CDA) [Ratcliff et al., 1992], and the STARDUST Cometary and Interstellar Dust Analyzer (CIDA) [Brownlee et al., 1995]. Auer [2001] has written an excellent review of the instrumentation and experimental techniques associated with in situ cosmic dust analysis.

[4] The majority of dust grains encountered by spacecraft range from 0.1 to $10 \mu \mathrm{m}$ in diameter. Within the solar system, dust grains become photoelectrically charged to typical potentials of 3-4 V with respect to charge neutrality [Kimura and Mann, 1998], corresponding to $2 \times 10^{-16} \mathrm{C}$ on a 1- $\mu$ m particle. The elemental composition of most solar system dust grains is approximately chondritic, although individual particles may have disproportionate contributions from specific minerals such as $\mathrm{Mg}$ silicates, FeNi metal, sulfides, phosphides, or carbonates [Brownlee, 1995]. Interstellar dust grains may show large deviations in isotopic and elemental composition as compared to solar system objects. Interstellar grains pass through the solar system at $25 \mathrm{~km} / \mathrm{s}$ relative to the sun, so impacts with spacecraft range from 0 to $50 \mathrm{~km} / \mathrm{s}$ depending on spacecraft location and trajectory. Encounters with other types of dust exhibit a similarly wide range of impact speeds.

[5] We recently developed a prototype model of a new in situ dust analyzer, called the Dustbuster, which has been described in detail [Austin et al., 2002]. As shown in Figure 1, ions produced by an impact on the target plate 


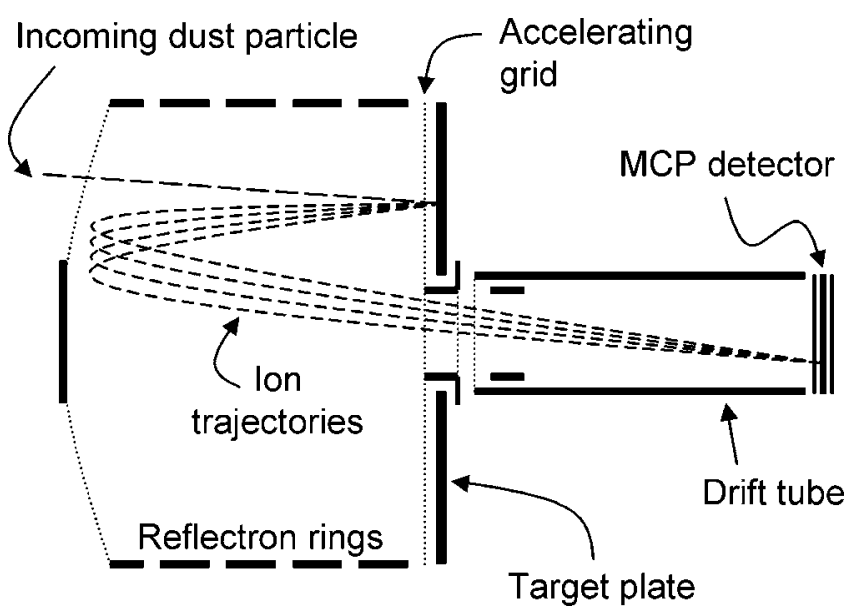

Figure 1. Design of the Dustbuster mass spectrometer (side view). Ions produced by the impact of a dust particle on the target plate are accelerated into the reflectron, through a drift tube and onto a microchannel plate (MCP) electron multiplier. The instrument has cylindrical symmetry and is $20 \mathrm{~cm}$ long and $10 \mathrm{~cm}$ in diameter.

are accelerated through a reflectron and onto a microchannel plate (MCP) detector or other suitable electron multiplier. The Dustbuster is considerably smaller and lighter than previous dust analyzers, and novel ion optics permit high mass resolution and a large sampling target area.

[6] Preliminary tests of the Dustbuster [Austin et al., 2002] used laser desorption ionization of copper metal and copperiron sulfide samples embedded in the target plate to simulate ionization from dust impacts. Typical mass resolution in these experiments ranged from $80-180(\mathrm{~m} / \Delta \mathrm{m})$ for isotopes of iron and copper. Although laser ionization is sufficiently similar to impact ionization to make a useful preliminary evaluation of the Dustbuster's performance, more realistic testing and calibration must involve impacts of microparticles resembling cosmic dust in their impact velocity, charge, size, and composition.

[7] Electrostatically accelerated microparticles in the laboratory are limited to either conducting materials or insulating materials coated with a conducting surface. Most minerals therefore are unsuitable for such impact experiments. In the current work, highly charged copper and iron microparticles, accelerated to $2-20 \mathrm{~km} / \mathrm{s}$ using a $2 \mathrm{MV}$ van de Graaff electrostatic accelerator, verify the Dustbuster's ability to analyze particles that approximate those of the space environment.

\section{Experimental Procedure}

[8] The principal components of the experimental setup, illustrated in Figure 2, include the vacuum system, the dust reservoir and charging needle, the van de Graaff ball, charge-sensitive electrodes along the length of the flight tube, and the Dustbuster time-of-flight mass spectrometer located at the end of the flight tube.

[9] The van de Graaff dust accelerator, originally built for NASA by Otto Berg, is similar to the dust accelerator built by Friichtenicht [1962]. Micron-sized iron and copper particles were placed in the dust reservoir, which was then evacuated.
A pulsed voltage applied to an electrode excited the particles, causing them to bounce around within the reservoir. Periodically a single particle left the reservoir through an aperture and became highly charged by contact with the charging needle. From there, the particle was accelerated by approximately $1.5 \mathrm{MV}$ into the flight tube and onto the Dustbuster. On average, $1-10$ particles per minute struck the Dustbuster impact plate. Because the spectrum acquisition time was only $10 \mu \mathrm{s}$, the probability of overlapping spectra from coincident particles was negligible.

[10] Three cylindrical electrodes within the flight tube allowed measurement of the charges and velocities of the accelerated particles. Each particle induced an image current as it passed through each cylinder. The resulting voltage pulses were combined, amplified, and recorded by an oscilloscope. Particle charge was calculated using the relation $q=\mathrm{CV}$, where $\mathrm{C}$ is the measured capacitance of the first cylinder electrode in the drift tube, and $\mathrm{V}$ is the magnitude of the induced voltage pulse. Particle velocity was determined from the distance between the other two electrodes and the time between those signals. Particle mass was found from $m=2 q \mathrm{~V}_{\mathrm{a}} / v^{2}$, where $\mathrm{V}_{\mathrm{a}}$ is the total accelerating voltage and $v$ is the measured particle velocity. The lower limit of charge sensitivity of the cylinder electrodes was approximately $1 \mathrm{fC}$, which resulted in a maximum observable velocity of $20-30$ $\mathrm{km} / \mathrm{s}$ and minimum observable mass of around $5 \times 10^{-18}$ $\mathrm{kg}$, based on typical surface charge densities of the accelerated metal particles. Charge is roughly proportional to surface area, and mass is proportional to volume, leading to an overall $v \propto r^{-1 / 2}$ relationship. As a result, smaller particles with less charge are accelerated to higher velocities than larger particles with more charge.

[11] The dust reservoir, accelerator, and flight tube were evacuated by a diffusion pump. Because this vacuum system was only able to maintain $3 \times 10^{-6}$ torr at the exit of the flight tube, the Dustbuster was mounted inside its own vacuum chamber, evacuated by a turbopump to $10^{-7}$ torr. The two vacuum systems were connected with a $1-\mathrm{cm}$ circular aperture, through which the charged particles left the flight tube and entered the Dustbuster chamber. The Dustbuster vacuum chamber included angled connection ports in order to observe particle impacts at angles of up to $25^{\circ}$ from the normal. A flexible bellows directed the particle beam to various locations on the target plate, spanning all radial distances from 1.5 to $4 \mathrm{~cm}$ from the instrument axis. The Dustbuster vacuum chamber was mounted on a large, custom-designed xyz translation/3-axis rotation stage that assisted in varying and measuring the angle and location of the particle impacts on the target plate.

[12] Particles used included wire-exploded copper with an average diameter of 100-150 nm (Argonide Nanomaterials), 1-micron copper (Sigma Chemical), and 0.1- to 3-micron (diameter) iron spheres produced by the mist reduction of iron carbonyl (General Aniline and Film Corp.). Initial experiments were carried out using iron particles alone. On the assumption that the composition of each particle could be deduced from its mass spectrum, the dust reservoir was filled with a mixture of copper and iron powder in later experiments.

[13] For all experiments the entrance grid and extraction grid of the Dustbuster were made of knitted tungsten (Kimball Physics) and electroformed nickel (Buckbee- 


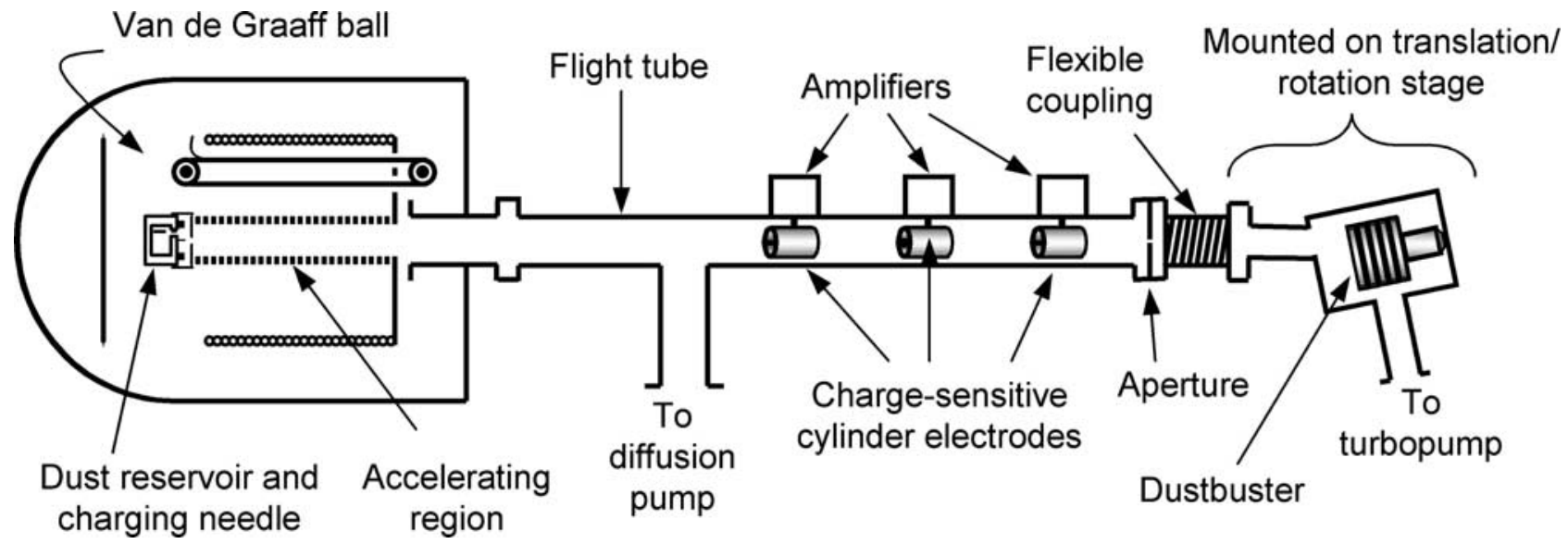

Figure 2. Experimental setup for hypervelocity microparticle impact studies. Micron-sized copper and iron spheres are charged in the van de Graaff ball, then individually accelerated through the flight tube and onto the Dustbuster mass spectrometer. Flight tube is approximately $5 \mathrm{~m}$ long.

Mears), respectively. The target plate was made out of 99.95\% pure tantalum (Alfa Aesar), sanded with 2400-grit silicon carbide sandpaper, degreased, cleaned, and sonicated in HPLC grade methanol.

[14] The Dustbuster was originally designed to use $4.8 \mathrm{kV}$ for ion extraction. In order to determine the possibility of operating the instrument using less power, a number of lower voltages were also tried, with all electrostatic potentials scaled proportionally. In addition, the voltage on the microchannel plate detector was independently varied from 700 to 900 volts per plate in order to determine the optimum conditions for observing the mass spectra. The signal from the microchannel plate detector was amplified with a $20 \mathrm{x}$ gain differentiating preamplifier (EG\&G Ortec VT120) and recorded using a $150 \mathrm{MHz}$ digital oscilloscope (Yokagawa 1520). Spectra were calibrated using the sodium and potassium $\left({ }^{39} \mathrm{~K}\right)$ peaks. Time-equals-zero points were not directly measured, but were extrapolated using the calibration points. A total of 750 spectra were recorded.

\section{Results}

[15] Figure 3 shows particle velocity as a function of mass for all particles studied. Measured particle velocities were generally between 1 and $10 \mathrm{~km} / \mathrm{s}$, although velocities up to $23 \mathrm{~km} / \mathrm{s}$ were observed. Uncertainties in velocity and charge measurements were approximately 5\%. Figure 4 shows the mass and charge of all detected particles. Also included are lines of constant surface charge density for ideal iron spheres in the range of the measured particles. The charge density of nearly all the particles in this experiment were at least an order of magnitude below the field desorption limit, $2 \times 10^{10} \mathrm{~V} / \mathrm{m}$, consistent with previous results using similar charging methods [Friichtenicht, 1962, 1964; Shelton et al., 1960]. Interestingly, the larger particles were charged to a fairly constant surface charge density $\left(5 \times 10^{-3} \mathrm{C} / \mathrm{m}^{2}\right)$, while the smallest particles follow more closely a curve of constant surface potential. During the experiments, a large number of particles struck the Dustbuster and triggered the recording electronics, but had too small a charge to be detected by the cylinder electrodes in the accelerator flight tube. Nonetheless, most of these particles generated interpretable mass spectra.

[16] Figures 5 and 6 show representative mass spectra for particles with defined and undefined velocities, respectively. Spectrum 5a, typical of roughly $20 \%$ of the impacts, shows only $\mathrm{Na}^{+}$and $\mathrm{K}^{+}$peaks with high mass resolution. Spectrum $5 \mathrm{~b}$ shows $\mathrm{H}^{+}$and unusually broad $\mathrm{Na}^{+}$and $\mathrm{K}^{+}$peaks. Spectrum $5 \mathrm{c}$ shows broad $\mathrm{Na}^{+}$and $\mathrm{K}^{+}$peaks along with a strong $\mathrm{Fe}^{+}$peak. Similarly broadened peaks appeared in about $15 \%$ of the spectra, but $\mathrm{Na}^{+}$and $\mathrm{K}^{+}$were the only species for which significant broadening occurred. In general, these peaks were broader for slower impact velocities. Impact angle, which was varied from $0^{\circ}$ to $23^{\circ}$, had no noticeable effect on the amount of peak broadening. In many spectra, the number of ions observed from a given $\mathrm{m} / \mathrm{z}$ exceeded the input voltage limit of the signal amplification

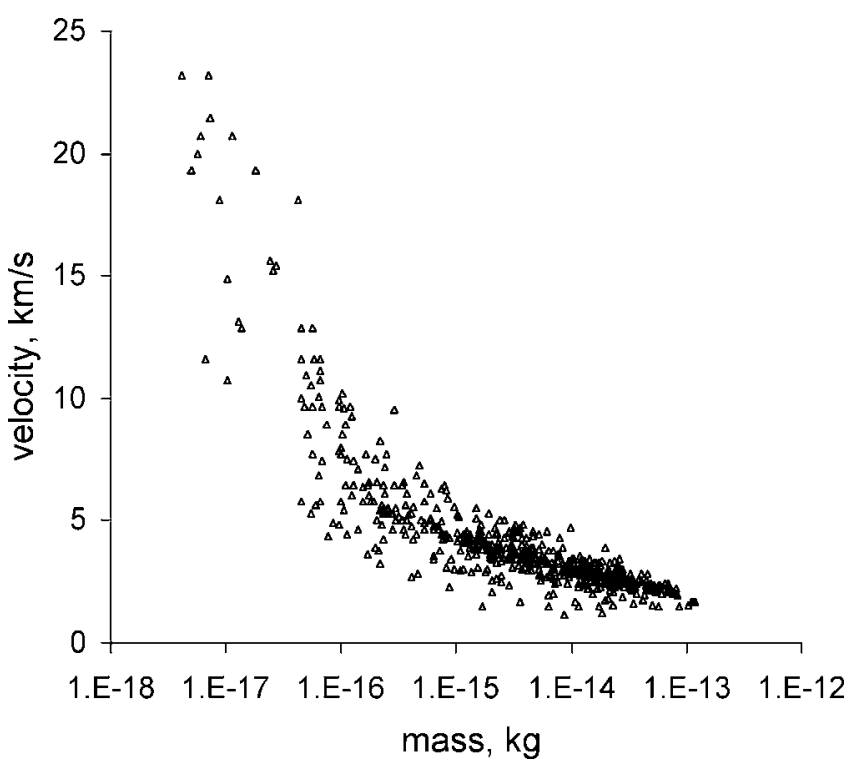

Figure 3. Velocities and masses of accelerated particles as measured by the charge-sensitive electrodes in the accelerator flight tube. 


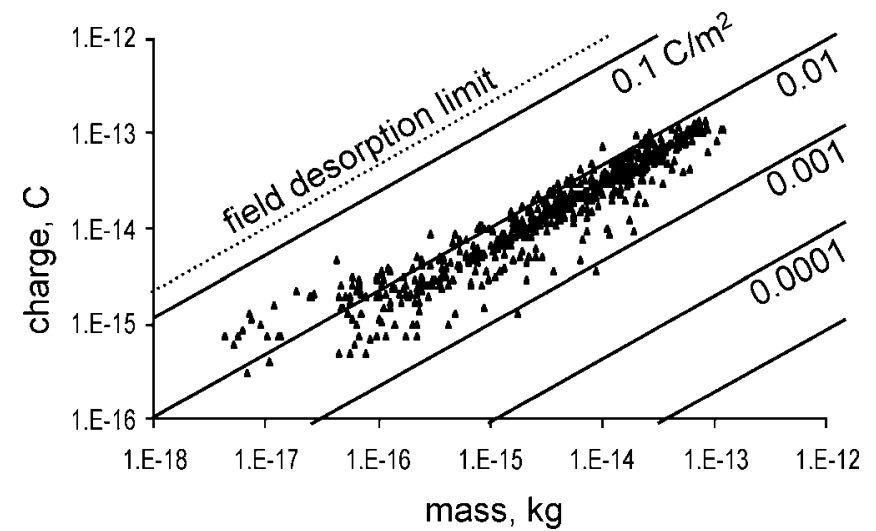

Figure 4. Charge vs. mass for accelerated iron and copper particles used in this study. Diagonals are lines of constant surface charge density, in $\mathrm{C} / \mathrm{m}^{2}$, for spherical iron particles. Uppermost line is the ion emission (ion evaporation) limit for iron.

or recording electronics, producing the peak clipping seen in spectra $5 \mathrm{c}$ and $5 \mathrm{~d}$. Spectrum $5 \mathrm{~d}$ shows peaks corresponding to $\mathrm{H}^{+}, \mathrm{C}^{+}, \mathrm{O}^{+}, \mathrm{Na}^{+}, \mathrm{K}^{+}, \mathrm{Fe}^{+}$, and a peak at $m / z=40$, which was not positively identifiable. Spectra $5 \mathrm{e}$ and $5 \mathrm{f}$
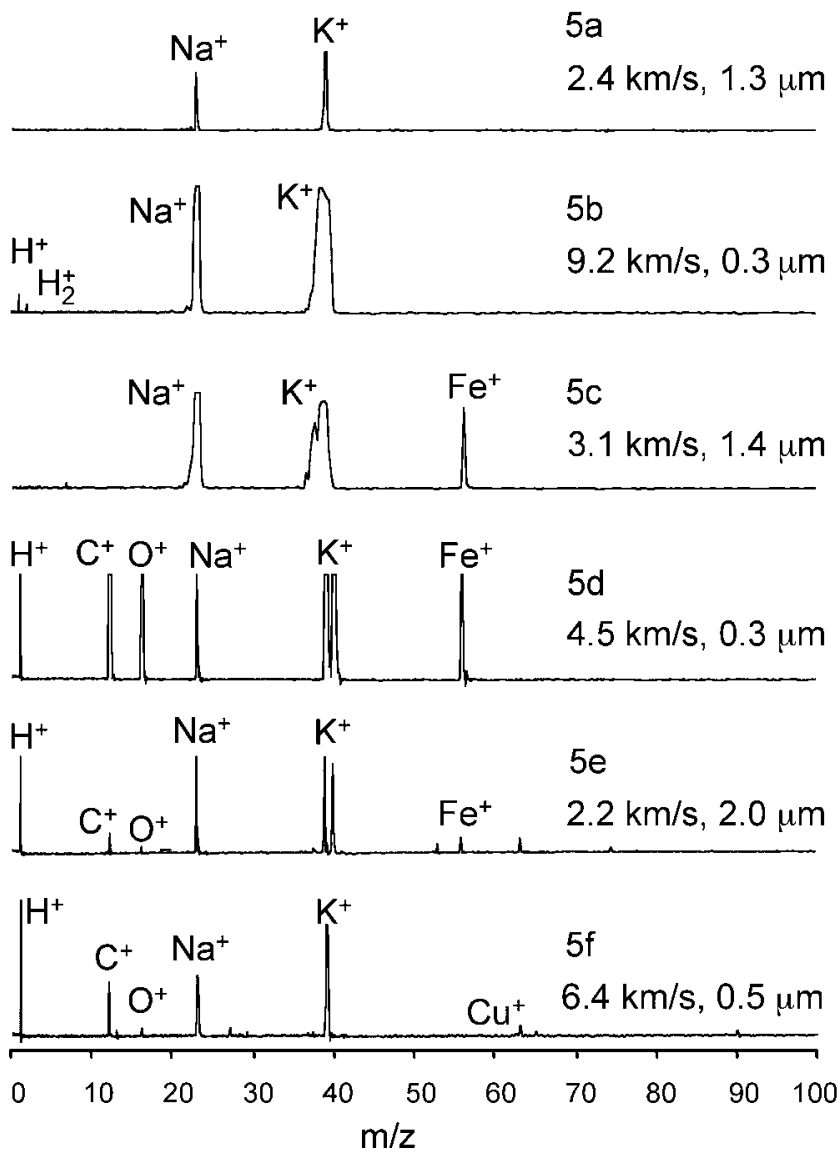

Figure 5. Typical mass spectra produced by microparticle impacts on the Dustbuster. The first four spectra are from iron particles; the last is a copper particle. Next to each spectrum is the velocity and size of the particle producing the spectrum. Vertical axis for each spectrum is signal intensity (arbitrary units).

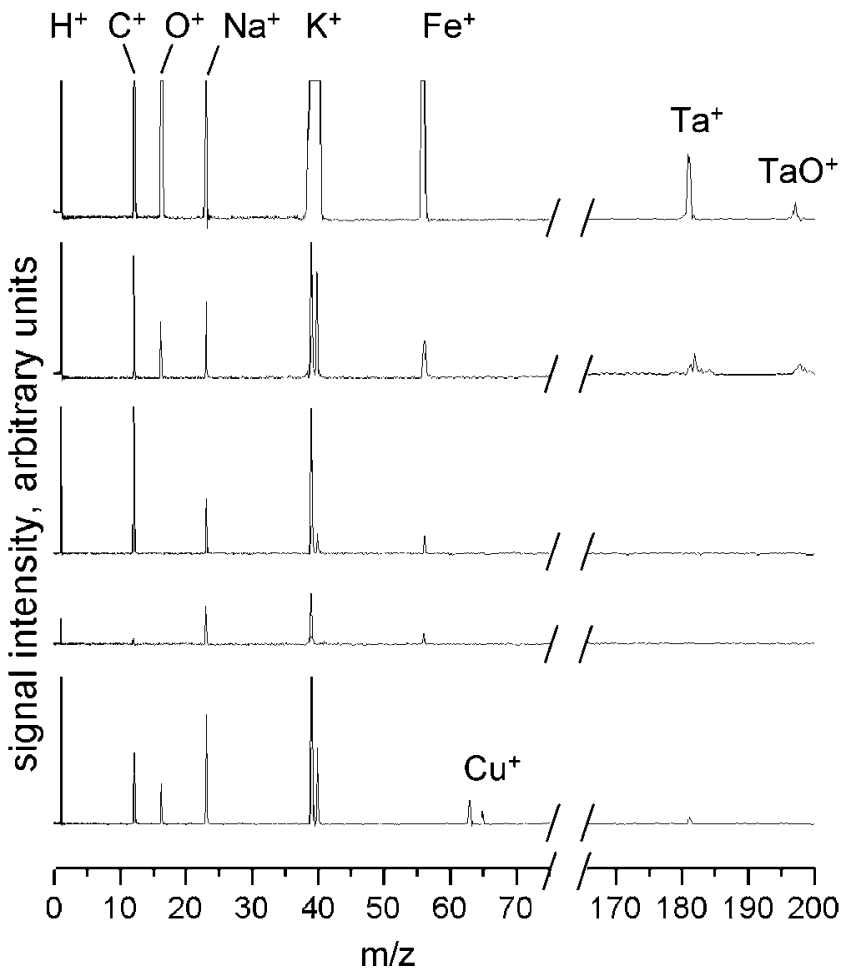

Figure 6. Spectra from particles with unknown mass and velocity. The first four spectra are iron impacts and the last is a copper particle. No peaks were observed in any spectra between $\mathrm{m} / \mathrm{z}=70$ and $\mathrm{m} / \mathrm{z}=180$. Vertical axis for each spectrum is signal intensity.

contain similar peaks with varying intensities. Figure 6 shows representative spectra produced by particles whose masses and velocities were beyond the detection limits of the cylinder electrodes. $\mathrm{Ta}^{+}$and $\mathrm{TaO}^{+}$from the target plate material are observed in some spectra. Table 1 enumerates all the mass peaks present in at least some of the spectra, and shows the ranges of mass resolution $(m / \Delta m)$ for several species. For lighter species, particularly hydrogen, mass resolution was limited by the speed of the recording oscilloscope rather than by a distribution of ion arrival times.

\section{Discussion}

[17] In general, faster particles produced a larger number of ions, consistent with previous results [Friichtenicht, 1964]. As expected, neither the incident angle of impact nor the location of the impact on the target plate produced any measurable difference in the mass resolution or other features of the resulting spectra. A statistical analysis of all the spectra failed to detect any correlation between the presence or absence of any specific peaks and the velocities, masses, kinetic energies, or momenta of the impacting particles.

[18] Several correlations exist relating the specific spectral peaks observed. For instance, $\mathrm{Ta}^{+}(\mathrm{m} / \mathrm{z}=181), \mathrm{TaO}^{+}$ $(m / z=197)$, and a peak at $m / z=40$ appear only in those spectra in which carbon and hydrogen are both present. $\mathrm{C}^{+}$ is always present, and $m / z=40$ is generally present in those 
Table 1. Summary of All Peaks Present in at Least Three Impact Spectra $^{\mathrm{a}}$

\begin{tabular}{cccc}
\hline m/z & $\begin{array}{c}\text { Percent of Spectra } \\
\text { With Given Peak }\end{array}$ & $\begin{array}{c}\text { Peak } \\
\text { Assignment }\end{array}$ & $\begin{array}{c}\text { Mass } \\
\text { Resolution }\end{array}$ \\
\hline 1 & 77 & $\mathrm{H}^{+}$ & $20-25$ \\
2 & 15 & $\mathrm{H}^{+}$ & \\
3 & 0.6 & $\mathrm{H}^{+}$ & \\
7 & 8 & $\mathrm{Li}^{+}$ & $50-150$ \\
12 & 59 & $\mathrm{C}^{+}$ & \\
13 & 0.7 & $\mathrm{CH}^{+}$ & \\
16 & 28 & $\mathrm{O}^{+}$ & \\
23 & 98 & $\mathrm{Na}^{+}$ & $5-200$ (typically 100) \\
27 & 1.5 & $\mathrm{Al}^{+}$ & \\
28 & 1.0 & $\mathrm{CO}^{+} \mathrm{or} \mathrm{Si}^{+} ?$ & $\mathrm{~K}^{+}$ \\
$39(41)$ & 99.5 & $\mathrm{Ar}^{+}, \mathrm{SiC}^{+}$or Ca $^{+} ?$ & $5-200$ (typically 100) \\
40 & $37^{\mathrm{b}}$ & $\mathrm{Fe}^{+}$ & $150-350$ \\
$56(54)$ & 16 & $\mathrm{Cu}^{+}$ & $150-350$ \\
$63(65)$ & 8 & $\mathrm{Ta}^{+}$ & $300-600$ \\
181 & 9 & $\mathrm{TaO}^{+}$ & \\
197 & 6 & & \\
\hline
\end{tabular}

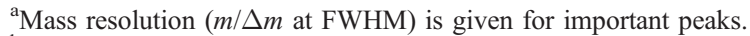

${ }^{b}$ Peaks may have been obscured by broad potassium lines; hence the occurrence of this peak may be more frequent than indicated.

spectra containing an oxygen ion peak. $\mathrm{H}_{2}^{+}$appears only when $\mathrm{H}^{+}$is present, but never when $\mathrm{Fe}^{+}$or $\mathrm{Cu}^{+}$are present.

[19] In most spectra, peak intensities corresponding to minor isotopes, such as ${ }^{41} \mathrm{~K}^{+}(6.7 \%$ natural abundance $)$ and ${ }^{54} \mathrm{Fe}^{+}(5.8 \%$ natural abundance $)$, were smaller than expected. In a few spectra, minor isotopes were absent. Several factors may be responsible for this effect. First, microchannel plate detectors are known to have nonlinear response to isotopes in some situations [Giudicotti et al., 1993; Odom and Schueler, 1990]. Although MCPs have been used for careful isotope ratio measurements, corrections must be made for gain saturation and other effects [Maechling et al., 1995]. In the current experiment minor isotope peaks may have been truncated or improperly sampled [Kang and Beauchamp, 1985]. Another possibility is that peaks such as $\mathrm{m} / \mathrm{z}=56$ may have had, in addition to ${ }^{56} \mathrm{Fe}^{+}$, an additional contribution from a contaminant such as $\mathrm{CaO}^{+}$, reducing the expected intensity of other iron isotopes with respect to the principal peak at 56 . If this is the case, the $\mathrm{m} / \mathrm{z}=40$ peak may be $\mathrm{Ca}^{+}$, although calcium was not expected to be a significant contaminant in this experiment, and no ${ }^{44} \mathrm{Ca}^{+}$peak was detected. Insufficient information exists to indicate which of these possible scenarios is responsible for the anomalous intensities of minor isotope peaks in the spectra. For a flight instrument, however, faster recording electronics and a better ion detector would reduce this obstacle to isotope ratio analysis.

[20] Sodium and potassium ion peaks were observed in nearly all spectra. In many of the impacts, these were the only observed ion species. Because of their low ionization potentials (5.139 eV for sodium, $4.341 \mathrm{eV}$ for potassium) and prevalence on metal surfaces, these alkali ion contaminants commonly appear in mass spectrometry involving surface ionization.

[21] Nonmetal ion peaks $\left(\mathrm{H}^{+}, \mathrm{C}^{+}, \mathrm{O}^{+}\right.$, etc. $)$were observed despite not being predicted by impact ionization models [see, e.g., Hornung et al., 2000]. Hydrogen, carbon, and oxygen have relatively high ionization potentials (13.598, 11.260 , and $13.618 \mathrm{eV}$, respectively) and typical $\mathrm{C}-\mathrm{H}$ bond strengths in hydrocarbons are $4 \mathrm{eV}$. Similar peaks have been observed in previous impact experiments, although their source is unclear. Roy [1975] studied impacts of $2-32 \mathrm{~km} / \mathrm{s}$ iron microparticles on a tantalum surface. He observed $\mathrm{H}^{+}$, $\mathrm{H}_{2}{ }^{+}, \mathrm{C}^{+}$and a peak at $\mathrm{m} / \mathrm{z}=28$ which he attributed to $\mathrm{Si}^{+}$ for particles above $9 \mathrm{~km} / \mathrm{s}$. His instrument did not have sufficient resolution to separate ${ }^{39} \mathrm{~K}^{+}$from any peak at $\mathrm{m} / \mathrm{z}=$ 40 , if present. His analysis was based on averaged spectra, prohibiting correlations between peaks in individual spectra. A $9 \mathrm{~km} / \mathrm{s}$ impact producing $0.4 \mathrm{eV}$ per nucleon does not have sufficient kinetic energy to atomize and ionize species with such high bond dissociation and ionization energies. According to hydrocode calculations by Hornung et al. [2000], even at $80 \mathrm{~km} / \mathrm{s}$ only $0.3 \%$ of the oxygen in a $\mathrm{SiO}_{2}$ particle will be ionized. In high velocity studies, Ratcliff and Allahdadi [1996] observed $\mathrm{H}^{+}$and $\mathrm{C}^{+}$peaks in their mass spectrum of a $94 \mathrm{~km} / \mathrm{s}$ boron carbide particle impacting a silver-plated aluminum target. They attributed these peaks to hydrocarbon (pump oil) contamination, but gave no explanation for the lack of hydrocarbon fragment peaks.

[22] Although the energy required to produce free $\mathrm{H}, \mathrm{C}$, and $\mathrm{O}$ ions is much higher than the impact energy, the presence of these peaks may be explained by the theory proposed by Novikov et al. [1988] and discussed by Sysoev et al. [1992, 1997] and McDonnell et al. [2001]. They postulate that an electric discharge occurs between the charged particle and target surface immediately before impact. According to their model, when the charged microparticle is within approximately $1-2 \mu \mathrm{m}$ of the surface, the high electric field causes electrons to desorb from the target surface and strike the particle, causing electron-induced desorption and ionization. In addition, a small spot on the particle surface is strongly heated, causing thermal desorption and ionization of such species as $\mathrm{Na}$ and $\mathrm{K}$. Under laboratory accelerator conditions the electron-emission model should have a loose dependence on impact velocity with the expectation that faster particles, with generally fewer charges, would result in fewer electron-generated ions. However, the velocity limits for this process have not been explored. In addition, particles encountered in space are not subject to the same mass-charge parameter limitations as electrostatically accelerated particles in a laboratory. It is likely that ions formed in a discharge that occurs as a charged particle approaches a target would be accelerated back onto the impact plate, producing additional ionization in a SIMS-like mechanism. In the present work, both the accelerated particles and the impact plate likely had surface contamination of pump oil, which in this case consisted of tetramethyltetraphenyltrisiloxane. The $\mathrm{O}^{+}$peak observed could be caused by oxygen from the pump oil, oxides on the particle surface, or by adsorbed gases, dissociated and ionized by field-emitted electrons prior to impact.

[23] If ionization is enhanced at a particle surface owing to electron emission or other surface effects (such as have been suggested by Hornung et al. [2000]), the quantity and type of ions formed in any given impact may not be representative of the bulk particle composition. Surface ionization effects must therefore be taken into account in interpreting spectra both from laboratory impacts and also from impacts of particles in space.

[24] Both in the Dustbuster and other instruments, electron-induced ionization may also occur as particles pass 

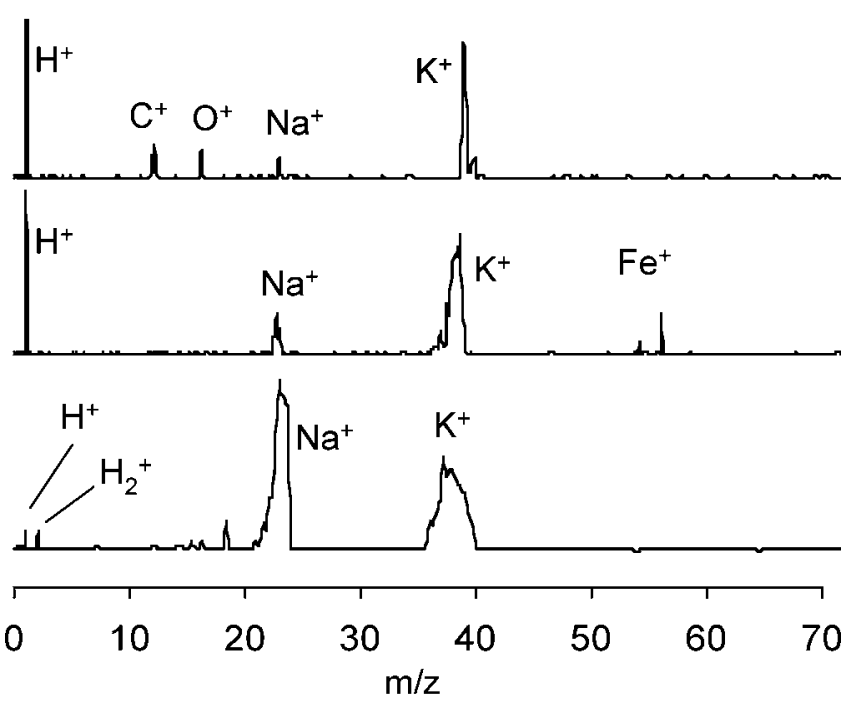

Figure 7. Spectra from three 2.6-micron iron particles impacting at $2.1 \mathrm{~km} / \mathrm{s}$. Vertical axis in each spectrum is signal intensity.

through grids and come close to a grid wire. For instance, in the Dustbuster, $15 \%$ of $1-\mu \mathrm{m}$ particles pass within $2 \mu \mathrm{m}$ of a grid wire before they reach the impact target. Inconsistent electrical interactions may produce different mass spectra for otherwise identical particles. Similarly, field emission of electrons from the target plate will vary with the microscale topology of the impact surface, possibly affecting the quantity and nature of ions produced in impacts of otherwise identical particles. Both of these effects have implications for in situ flight instruments. Impact cratering of a target plate, which can be extensive in some dust environments, would change the ionizing characteristics of a target plate over time. Also, particle charge measurements are necessary in order to predict the specific ionization mechanism responsible for a given spectrum.

[25] The presence or absence of certain mass peaks shows a great deal of variability between similar microparticles. This variability is sufficient to obscure correlations that might exist between measured particle parameters and spectral features. For instance, given two particles identical in mass, composition, charge, and velocity, one may produce significant $\mathrm{C}, \mathrm{H}$, and $\mathrm{O}$ ion peaks, while such peaks might be completely absent from the spectrum of the other particle. Figure 7 shows an example of this behavior. Three 2.6-micron iron particles impacting normal to the target, at $2.1 \mathrm{~km} / \mathrm{s}$, and in the same region of the target plate, produce three different spectra. The origin of this variation in spectra is not clear, but illustrates the dependence of the ionization mechanism on one or more unmeasured variables. These variables may pose significant limitations on estimates of elemental composition from any cosmic dust analyzer of this type.

[26] A few other unusual observations deserve comment. As seen in Figures 5-7, some spectra exhibit unusually broad $\mathrm{Na}^{+}$and $\mathrm{K}^{+}$peaks, although other ion species seem unaffected. Because the Dustbuster uses a reflectron, it is unlikely that these broad peaks are caused by differences in the initial kinetic energies of the ions. More likely, these ions are produced over a longer timescale than the other ions. Knabe and Krueger [1982], who observed similarly broadened alkali ion peaks, suggested that the peak broadening is the result of thermal emission. For broadened peaks from slower impacts $(1.5-2.5 \mathrm{~km} / \mathrm{s})$, the peak widths correspond to roughly $300 \mathrm{~ns}$, while faster impacts produce less broadening. It is possible that these broad peaks arise from particles that were heated by electron bombardment while passing through the acceleration grid. Such particles would emit sodium and potassium ions all the way through the acceleration region. Computer simulations of this situation using SIMION ion trajectory software [Dahl, 2000] show that only those ions emitted within $300-400 \mu \mathrm{m}$ of the target plate would have the correct energy and trajectory to reach the ion detector. The combination of being formed early and gaining less kinetic energy would result in an arrival time of about $0-300$ ns earlier for these ions, consistent with observations.

\section{Conclusions}

[27] Mass resolution from particle impacts is equal to or better than previous Dustbuster results using laser desorption ionization. Although a few mass spectra show unusual peak broadening, the majority have high mass resolution, showing that the Dustbuster functions well as a compact time-of-flight mass spectrometer for in situ cosmic dust analysis. Peaks such as $\mathrm{C}^{+}, \mathrm{H}^{+}$, and $\mathrm{O}^{+}$may not be representative of particle composition, but may provide useful clues as to what is taking place on the surfaces of dust grains. Impact mass spectra still provide bulk composition, although accuracy may be limited by the complicated ionization mechanism taking place in impacts involving charged microparticles.

[28] Acknowledgments. The authors thank NASA's Planetary Instrument Definition and Development Program (PIDDP) for financial support. Additional financial support for the early stages of this research came from the Beckman Institute at Caltech. Contribution 8870, Division of Geological and Planetary Sciences, California Institute of Technology, Pasadena, CA 91125.

\section{References}

Auer, S., Instrumentation, in Interplanetary Dust, edited by E. Grün et al., pp. 385-444, Springer-Verlag, Berlin, 2001.

Austin, D. E., T. J. Ahrens, and J. L. Beauchamp, Dustbuster: A compact impact-ionization time-of-flight mass spectrometer for in situ analysis of cosmic dust, Rev. Sci. Instrum., 73(1), 185-189, 2002.

Brownlee, D. E., The elemental composition of interplanetary dust, in Physics, Chemistry, and Dynamics of Interplanetary Dust, 150th Colloquium of the International Astronomical Union, edited by B. A. S. Gustafson and M. S. Hanner, pp. 261-264, Astron. Soc. of the Pac., San Francisco, Calif., 1995

Brownlee, D. E., D. Burnett, B. Clark, M. S. Hanner, F. Horz, J. Kissel, R. Newburn, S. Sandford, Z. Sekanina, P. Tsou, and M. Zolensky, STARDUST: Comet and interstellar dust sample return mission, in Physics, Chemistry, and Dynamics of Interplanetary Dust, 150th Colloquium of the International Astronomical Union, edited by B. A. S. Gustafson and M. S. Hanner, pp. 223-226, Astron. Soc. of the Pac., San Francisco, Calif., 1995

Dahl, D. A., SIMION 3D, Idaho Natl. Eng. and Environ. Lab., Idaho Falls, Idaho, 2000

Friichtenicht, J. F., Two-million-volt electrostatic accelerator for hypervelocity research, Rev. Sci. Instrum., 33(2), 209-212, 1962.

Friichtenicht, J. F., Micrometeoroid simulation using nuclear accelerator techniques, Nucl. Instrum. Methods, 28, 70-78, 1964.

Giudicotti, L., M. Bassan, R. Pasqualotto, and A. Sardella, Simple analytical model of gain saturation in microchannel plate devices, Rev. Sci. Instrum., 65(1), 247-258, 1993. 
Grün, E., Dust measurements in the outer solar system, in Asteroids, Comets, Meteors 1993, Int. Astron. Union Sympos. Ser., vol. 160, pp. $367-$ 380, Kluwer Acad., Norwell, Mass., 1994.

Hansen, D. O., Mass analysis of ions produced by hypervelocity impact, Appl. Phys. Lett., 13(3), 89-91, 1968.

Hornung, K., and J. Kissel, On shock wave impact ionization of dust particles, Astron. Astrophys., 291, 324-336, 1994.

Hornung, K., Y. G. Malama, and K. S. Kestenboim, Impact vaporization and ionization of cosmic dust particles, Astrophys. Space Sci., 274, $355-$ 363, 2000.

Kang, H., and J. L. Beauchamp, Pulsed laser evaporation and ionization of solid metal targets. Implications for studying the gas-phase reactions of laser-generated atoms and ions, J. Phys. Chem., 89, 3364-3367, 1985.

Kimura, H., and I. Mann, The electric charging of interstellar dust in the solar system and consequences for its dynamics, Astrophys. J., 499, 454462, 1998.

Kissel, J., et al., Composition of Comet Halley dust particles from Vega observations, Nature, 321, 280-282, 1986.

Knabe, F. R., and F. R. Krueger, Ion formation from alkali iodide solids by swift dust particle impact, Z. Naturforsch., 37a, 1335-1340, 1982

Maechling, C. R., S. J. Clemett, and R. N. Zare, 13C/12C ratio measurements of aromatic molecules using photoionization with TOF mass spectrometry, Chem. Phys. Lett., 241, 301-310, 1995.

McDonnell, J. A. M., E. D. Aguti, and M. J. Willis, Pre-contact microdischarge from charged particulates, in Proceedings of the 7th Spacecraft Charging Technology Conference, 23-27 April 2001, pp. 423-427, Eur. Space Agency, Paris, 2001.

Novikov, L. S., N. D. Semkin, V. S. Kulikauskas, S. M. Semenchuk, and V. P. Kiryukhin, Mass spectrometry of ions emitted in collisions of accelerated dust particles with target, Sov. Phys. Tech. Phys., 33(6), 680-682, 1988 .

Odom, R. W., and B. Schueler, Laser microprobe mass spectrometry: Ion and neutral analysis, in Lasers and Mass Spectrometry, edited by D. M. Lubman, pp. 103-137, Oxford Univ. Press, New York, 1990.

Ratcliff, P. R., and F. Allahdadi, Characteristics of the plasma from a $94 \mathrm{~km} / \mathrm{s}$ microparticle impact, Adv. Space Res., 17(12), 87-91, 1996.

Ratcliff, P. R., J. A. M. McDonnell, J. G. Firth, and E. Grün, The Cosmic Dust Analyzer, J. Brit. Interplanet. Soc., 45(9), 375-380, 1992.

Roy, N. L., Research Investigations of the Physical Interactions and Phenomena Associated With Hypervelocity Sub-micron Particles, 67 pp., TRW Syst. Group, Redondo Beach, Calif., 1975.

Shelton, H., C. D. Hendricks Jr., and R. F. Wuerker, Electrostatic acceleration of microparticles to hypervelocities, J. Appl. Phys., 31(7), 12431246,1960

Srama, R., and E. Grün, The dust sensor for Cassini, Adv. Space Res., 20(8), 1467-1470, 1997.

Sysoev, A. A., D. R. Bandura, and V. P. Ivanov, Mechanism of ionization in a low-velocity collision of charged microparticles, Sov. Tech. Phys. Lett., 18(8), 486-488, 1992.

Sysoev, A. A., V. P. Ivanov, T. V. Barinova (Komova), and Y. A. Surkov, Mass spectra formation from charged microparticles, Nuclear Instrum. Methods Phys. Res., Sect. B, 122, 79-83, 1997.

T. J. Ahrens, Division of Geological and Planetary Sciences, California Institute of Technology, Pasadena, CA 91125, USA.

D. E. Austin, Sandia National Laboratories, Albuquerque, NM 871851411, USA.

C. L. Bailey, J. E. Farnsworth, and H. L. K. Manning, Department of Physics, Concordia College, Moorhead, MN 56562, USA.

J. L. Beauchamp and R. L. Grimm, Division of Chemistry and Chemical Engineering, California Institute of Technology, 1200 East California Boulevard, Mail Code 127-72, Pasadena, CA 91125, USA. (jlbchamp@ caltech.edu) 